\title{
Evaluation of Different Insecticides and Biorationals Against Scale Insect, Hemilecanium imbricans (Green) Under Laboratory Condition
}

\author{
Prakash $^{1^{*}}$ and R.R. Patil ${ }^{2}$ \\ ${ }^{1}$ Department of Agricultural Entomology, Agriculture College, Vijayapura, \\ UAS, Dharwad-05, India \\ ${ }^{2}$ Director of Extension, UAS, Dharwad-05, Karnataka, India \\ *Corresponding author
}

\section{A B S T R A C T}

Key w o r d s
$\begin{aligned} & \text { Scale insect, } \\ & \text { Hemilecanium, } \\ & \text { Insecticides, Biorationals, } \\ & \text { Mortality, Fish Oil Rosin } \\ & \text { Soap (FORS) etc. }\end{aligned}$
Article Info
$\begin{aligned} & \text { Accepted: } \\ & \text { 08 June } 2018 \\ & \text { Available Online: } \\ & \text { 10 July } 2018\end{aligned}$

Keywords

Scale insect,

Hemilecanium

Insecticides, Biorationals,

Soap (FORS) etc.

\section{Introduction}

Scale insects are most important as agricultural pest of perennial plants and can cause serious damage to nut and fruit trees, woody ornamentals, forest vegetation, greenhouse plants and house plants. Damage is usually caused by removal of plant sap, toxins and the excretion of large quantities of honeydew with resultant growth of sooty mold fungi that cover leaf surfaces and reduce photosynthesis. The waxy covering of many species of scale insects protects them effectively from contact insecticides, which are only effective against crawlers. However,
Among 25 insecticides and biorationals evaluated with and without Fish Oil Rosin Soap $(5 \mathrm{ml} / \mathrm{l})$ against different instars of scale insect, Hemilecanium imbricans, the insecticides viz., buprofezin $25 \mathrm{SC} @ 1.25 \mathrm{ml} / \mathrm{l}$, chlorpyriphos $20 \mathrm{EC} @ 2 \mathrm{ml} / \mathrm{l}$, acephate 75 SP @ 1 g/l, lambda-cyhalothrin 5 EC @ 0.5 ml/1, profenophos 50 EC @ 2 $\mathrm{ml} / \mathrm{l}$ and dichlorvos $76 \mathrm{EC} @ 1 \mathrm{ml} / \mathrm{l}$ were recorded highest per cent mortality of scales under laboratory conditions. Irrespective of treatments imposed with and without FORS against different stages of scale insect, $H$. imbricans similar effects were produced with respect to mortality percentage with little variation. With respect to instar susceptibility, the first instars (crawlers) and second instars of $H$. imbricans fourth instars under laboratory conditions. 
branches and twigs. Furthermore, the leaves of the infested trees showed a signs of yellowing (Kondo and Michael, 2005). Hemilecanium imbricans (Green) has become an endemic pest for particularly in Navalur, Kelageri, Jogeyellapur and Mugad villages of Dharwad district in North Karnataka. Lack of effective timely management practices, presence of protective shell and prolific breeding habits lead to build up of large population and spreading from one infested garden to another. In this context, the present investigation was undertaken.

\section{Materials and Methods}

In vitro bioassay was carried out with 25 treatments and two replications in Completely Randomized Design (CRD) to evaluate the relative efficacy of different insecticides and biorationals with and without fish oil rosin soap (5 ml/l) against all stages of scale insect, H. imbricans.

The recommended dosage of insecticides was diluted in water to get the required concentration. Known numbers (100) of first instar crawlers, second, third, and fourth instar stages on mango twig were selected and spraying was done with the help of baby sprayer ensuring equal number of strokes to all the treatments. The mango twigs were provided with cotton vad to maintain the moisture content of the twig. Mortality was recorded at an interval of 24 hours for seven days. Observations were revealed on shrinkage, shriveling, and mortality of scales percentage was worked out.

\section{Results and Discussion}

The results on efficacy of various treatments against scale insect, $H$. imbricans on mango twigs recorded in terms of mortality caused at one, three, five and seven days after spray with and without FORS are presented in table 1-7.

\section{First instar}

The precount recorded at one day before spray indicated non-significant difference between various treatments.

\section{One day after spray}

There was a satisfactory significant reduction in scale insect in all the treatments except untreated control. Buprofezin 25 SC @ 1.25 $\mathrm{ml} / \mathrm{l}$ was significantly superior to all other treatments recording 91.10 per cent mortality. It was on par with chlorpyriphos 20 EC @ 2 $\mathrm{ml} / \mathrm{l}$, acephate $75 \mathrm{SP} @ 1 \mathrm{~g} / \mathrm{l}$, lambdacyhalothrin 5 EC @ $0.5 \mathrm{ml} / 1$, profenophos 50 EC @ $2 \mathrm{ml} / \mathrm{l}$, buprofezin $5.65 \mathrm{SC}+$ deltamethrin 0.72 EC @ $2.5 \mathrm{ml} / \mathrm{l}$ and dichlorvos 76 EC @ 1 ml/l. However, application of pongamia oil + tween 80 recorded lowest mortality of 69.51 per cent (Table 1).

Various insecticides when imposed without FORS exhibited effect similar to with FORS. Buprofezin 25 SC @ $1.25 \mathrm{ml} / \mathrm{l}$ was superior recording 90.50 per cent mortality and was on par with chlorpyriphos 20 EC @ 2 ml/l, acephate75 SP@1 g/l, lambda-cyhalothrin 5 EC @ 0.5 ml/l, profenophos 50 EC @ 2 ml/l and dichlorvos 76 EC @ 1 ml/l. Cotton oil + tween and sunflower oil + tween effected lower mortality $(70.00 \%$ and $72.50 \%$ ) (Table 2). Various insecticides used with or without FORS the mortality trend were similar to 1DAS even at 3, 5 and 7 days after spray.

\section{Second instar}

\section{One day after spray}

$\begin{array}{lllllll}\text { Buprofezin } & 25 & \mathrm{SC} & @ & 1.25 & \mathrm{ml} / \mathrm{l} & \text { was }\end{array}$ significantly superior to all other treatments by recording 56.51 per cent mortality. The next best treatments were chlorpyriphos 20 EC @ 2 $\mathrm{ml} / \mathrm{l}$, acephate 75 SP @ 1 g/l, lambda- 
cyhalothrin 5 EC @ $0.5 \mathrm{ml} / \mathrm{l}$, profenophos 50 EC @ 2 ml/l, buprofezin 5.65 SC + deltamethrin 0.72 EC @ $2.5 \mathrm{ml} / \mathrm{l}$ and dichlorvos 76 EC @ 1 ml/1. Least mortality was recorded in Lecanicillium lecani + tween 80 treatment $(11.51 \%)$ (Table 3 ).

Treatments without FORS also performed results similar to with FORS but lower mortality compared to with FORS treatment. However, buprofezin 25 SC @ $1.25 \mathrm{ml} / 1$ was superior by recording 51.50 per cent mortality but was on par with dichlorvos 76 EC @ 1 $\mathrm{ml} / \mathrm{l}$. The next best treatments were chlorpyriphos 20 EC @ 2 ml/l, acephate 75 SP @ $1 \mathrm{~g} / 1$ and lambda-cyhalothrin 5 EC @ 0.5 $\mathrm{ml} / \mathrm{l}$. Least mortality of 10.50 per cent was recorded in sunflower oil + tween 80 treatment (Table 4).

\section{Three days after spray}

The chemical buprofezin 25 SC @ $1.25 \mathrm{ml} / 1$ was significantly superior over all other treatments by recording 64.51 per cent mortality but was on par with lambdacyhalothrin 5 EC @ $0.5 \mathrm{ml} / 1$, dichlorvos 76 EC @ 1 ml/l, chlorpyriphos 20 EC @ 2 ml/l and acephate 75 SP @ $1 \mathrm{~g} / 1$. Least mortality of 15.00 per cent was recorded in the treatment, pongamia oil + tween 80 (Table 3$)$.

Use of different insecticides without FORS recorded similar results with FORS treatment though per cent mortality was less. Buprofezin 25 SC @ $1.25 \mathrm{ml} / \mathrm{l}$ recorded 66.00 per cent mortality but on par with acephate 75 SP @ 1 g/l, lambda-cyhalothrin 5 EC @ 0.5 ml/l dichlorvos 76 EC @ $1 \mathrm{ml} / \mathrm{l}$ and chlorpyriphos 20 EC @ 2 ml/l. Least mortality of 12.00 per cent was recorded in sunflower oil + tween 80 treatment (Table 4).

\section{Five days after spray}

The insecticide lambda-cyhalothrin 5 EC @ $0.5 \mathrm{ml} / 1$ significantly differed from all other treatments with 91.00 per cent mortality but was on par with acephate 75 SP @ 1 g/l, chlorpyriphos 20 EC @ $2 \mathrm{ml} / \mathrm{l}$ and dichlorvos 76 EC @ 1 ml/l. Cotton oil + tween 80 treatment recorded least mortality of 10.00 per cent (Table 3). Treatments imposed without FORS did not differ in their effects that were imposed with FORS though with lower mortality rate. Buprofezin 25 SC @ $1.25 \mathrm{ml} / 1$ was the top most treatment with 86.50 per cent mortality but was on par with dichlorvos 76 EC @ $1 \mathrm{ml} / \mathrm{l}$. This was followed by chlorpyriphos 20 EC @ 2 ml/l, acephate 75 SP @ 1 g/l, lambda-cyhalothrin 5 EC @ 0.5 ml/l and. Sunflower oil + tween 80 treatment effected least mortality of 13.00 per cent (Table 4).

\section{Seven days after spray}

Buprofezin $\quad 25 \quad \mathrm{SC} \quad @ \quad \begin{array}{llll}1.25 & \mathrm{ml} / \mathrm{l} & \text { was }\end{array}$ significantly superior to all treatments with 99.00 cent per cent mortality. The next best treatments were chlorpyriphos 20 EC @ 2 $\mathrm{ml} / 1$, acephate 75 SP @ 1 g/l, lambdacyhalothrin 5 EC @ $0.5 \mathrm{ml} / 1$ and dichlorvos 76 EC @ 1 ml/l. Least mortality of 17.00 per cent was recorded in Pongamia oil + tween 80 treatment (Table 3). Insecticides when imposed without FORS did not differ much though they produced less mortality than with FORS treatment. Buprofezin 25 SC @ 1.25 $\mathrm{ml} / \mathrm{l}$ was significantly superior effecting 95.50 per cent mortality but was on par with lambdacyhalothrin 5 EC @ 0.5 ml/l, chlorpyriphos 20 EC @ 2 ml/l and dichlorvos 76 EC @ 1 ml/l. Least mortality of 14.00 per cent was recorded in the treatment, sunflower oil + tween 80 (Table 4).

\section{Third instar}

\section{One day after spray}

Acephate 75 SP $\quad @ \quad 1 \quad$ g/l $\quad$ performed significantly superior to all other treatments by recording 50.50 per cent mortality. This was 
on par with chlorpyriphos 20 EC @ $2 \mathrm{ml} / \mathrm{l}$, buprofezin 25 SC @ $1.25 \mathrm{ml} / \mathrm{l}$, lambdacyhalothrin 5EC @ $0.5 \mathrm{ml} / \mathrm{l}$ and dichlorvos 76 EC @ $1 \mathrm{ml} / \mathrm{l}$. Least mortality (8\%) was recorded in cotton seed oil + tween 80 treatment (Table 5). Results related to treatments without FORS on third instar revealed less mortality compared to with FORS treatment. Lambda-cyhalothrin 5 EC @ $0.5 \mathrm{ml} / \mathrm{l}$ treatment was significantly superior to other treatments by recording 48.00 per cent mortality but was on par with chlorpyriphos 20 EC @ 2 ml/l, acephate 75 SP @ 1 g/l, buprofezin $25 \mathrm{SC} @ 1.25 \mathrm{ml} / \mathrm{l}$ and dichlorvos 76 EC @ 1 ml/l. Least mortality (10\%) was recorded in the treatment sunflower seed oil + tween 80 (Table 6).

\section{Three days after spray}

Dichlorvos 76 EC @ 1 ml/l was superior with 77.50 per cent mortality but was on par with lambda-cyhalothrin 5 EC @ $0.5 \mathrm{ml} / \mathrm{l}$. The next best treatments were buprofezin 25 SC @ 1.25 $\mathrm{ml} / \mathrm{l}$, chlorpyriphos $20 \mathrm{EC} @ 2 \mathrm{ml} / \mathrm{l}$, acephate 75 SP @ 1 g/l and profenophos 50 EC @ 2 $\mathrm{ml} / \mathrm{l}$. Least mortality (9\%) was recorded in the treatment sunflower oil + tween 80 (Table 5).

Insecticides treatments without FORS also produced similar results as that of with FORS but with lower mortality.

Dichlorvos 76 EC @ $1 \mathrm{ml} / \mathrm{l}$ was superior by recording 70.50 per cent mortality but was on par with lambda-cyhalothrin 5 EC @ $0.5 \mathrm{ml} / \mathrm{l}$, chlorpyriphos $20 \mathrm{EC} @ 2 \mathrm{ml} / \mathrm{l}$, acephate $75 \mathrm{SP}$ @ $1 \mathrm{~g} / \mathrm{l}$, profenophos $50 \mathrm{EC}$ @ $2 \mathrm{ml} / \mathrm{l}$ and buprofezin $25 \mathrm{SC} @ 1.25 \mathrm{ml} / 1$. Least mortality $(12 \%)$ was recorded in the treatment pongamia oil + tween 80 (Table 6).

\section{Five days after spray}

Lambda-cyhalothrin 5 EC @ 0.5 ml/l was significantly superior to all other treatments with 86.00 per cent mortality but on par with buprofezin 25 SC @ $1.25 \mathrm{ml} / \mathrm{l}$. The next best treatments were chlorpyriphos 20 EC @ 2 $\mathrm{ml} / \mathrm{l}$, acephate $75 \mathrm{SP} @ 1 \mathrm{~g} / \mathrm{l}$, profenophos 50 EC @ $2 \mathrm{ml} / \mathrm{l}$ and dichlorvos 76 EC @ $1 \mathrm{ml} / \mathrm{l}$. Least mortality $(10.50 \%)$ was recorded in the treatment sunflower oil + tween 80 (Table 5).

Results without FORS exercised similar effects to that of with FORS on per cent mortality. Buprofezin 25 SC @ $1.25 \mathrm{ml} / 1$ topped with 79.00 per cent mortality and was on par with dichlorvos 76 EC @ 1 ml/l.

The next best treatments were chlorpyriphos 20 EC @ 2 ml/1, lambda-cyhalothrin 5 EC @ $0.5 \mathrm{ml} / \mathrm{l}$, acephate $75 \mathrm{SP} @ 1 \mathrm{~g} / \mathrm{l}$ and profenophos 50 EC @ 2 ml/l. Least mortality $(13.50 \%)$ was recorded in the treatment sunflower oil + tween 80 (Table 6).

\section{Seven days after spray}

The chemical insecticide buprofezin 25 SC @ $1.25 \mathrm{ml} / \mathrm{l}$ was significantly superior to all other treatments with 92.50 per cent mortality. The next best treatments were lambda-cyhalothrin 5 EC @ $0.5 \mathrm{ml} / \mathrm{l}$, acephate 75 SP @ $1 \mathrm{~g} / \mathrm{l}$, chlorpyriphos 20 EC @ $2 \mathrm{ml} / \mathrm{l}$ and dichlorvos 76 EC @ 1 ml/l. Least mortality (11.00\%)was recorded in the treatment sunflower oil + tween 80 (Table 5).

Insecticides imposed without FORS produced similar to those with FORS though with less mortality. Buprofezin $25 \mathrm{SC} @ 1.25 \mathrm{ml} / \mathrm{l}$ was significantly superior to all other treatments with 88.50 per cent mortality but was on par with chlorpyriphos 20 EC @ $2 \mathrm{ml} / \mathrm{l}$ and lambda-cyhalothrin 5 EC @ $0.5 \mathrm{ml} / \mathrm{l}$. The next best treatments were acephate 75 SP @ $1 \mathrm{~g} / 1$ and dichlorvos76 EC @ 1 ml/l.

Least mortality (14.50\%) was recorded in sunflower oil + tween 80 treatment (Table 6). 


\section{Fourth instar}

\section{One day after spray}

The chemical insecticide dichlorvos 76 EC @ $1 \mathrm{ml} / \mathrm{l}$ was significantly superior to all other treatments with 48.00 per cent mortality but on par with acephate 75 SP @ $1 \mathrm{~g} / \mathrm{l}$. The next best treatments were buprofezin 25 SC @ 1.25 ml/l, chlorpyriphos 20 $\quad$ EC @ 2 ml/l profenophos 50 EC @ $2 \mathrm{ml} / \mathrm{l}$ and lambdacyhalothrin 5 EC@0.5 ml/l. The treatment Neemazal treatment@15000 ppm recorded only 6.00 per cent mortality (Table 7).

Trend was similar when insecticides were applied without FORS. Buprofezin 25 SC @ $1.25 \mathrm{ml} / \mathrm{l}$ was superior among the various treatments by recording 35.50 per cent mortality and was on par with chlorpyriphos 20 EC @ 2 ml/l, acephate 75 SP @ 1 g/l, profenophos $50 \mathrm{EC} @ 2 \mathrm{ml} / \mathrm{l}$ and dichlorvos 76 EC @ 1 ml/l. Least mortality (9.00\%) was recorded in pongamia oil + tween 80 treatment (Table 8).

\section{Three days after spray}

The chemical insecticide buprofezin 25 SC @ $1.25 \mathrm{ml} / 1$ was significantly superior to all other treatments with 67.50 per cent mortality but was on par with acephate 75 SP @ $1 \mathrm{~g} / \mathrm{l}$ and dichlorvos76 EC @ $1 \mathrm{ml} / \mathrm{l}$. The next best treatments were chlorpyriphos 20 EC @ 2 $\mathrm{ml} / \mathrm{l}$, profenophos 50 EC @ $2 \mathrm{ml} / \mathrm{l}$ and lambda-cyhalothrin $\quad 5 \quad$ EC $\quad @ \quad 0.5 \quad \mathrm{ml} / 1$. However, least mortality (8.50\%) was recorded in the treatment pongamia oil + tween 80 (Table 7).

Treatments imposed without FORS exercised similar effects to that when treated with FORS. Buprofezin $25 \mathrm{SC} @ 1.25 \mathrm{ml} / \mathrm{l}$ was superior recording 50.50 per cent mortality but was on par with acephate 75 SP @ 1 g/l, chlorpyriphos 20 EC @ 2 ml/l, lambda- cyhalothrin 5 EC @ $0.5 \mathrm{ml} / \mathrm{l}$, profenophos 50 EC @ $2 \mathrm{ml} / 1$ and dichlorvos 76 EC @ 1 ml/l. The least mortality of 11.50 per cent was recorded in sunflower oil + tween 80 treatment (Table 8).

\section{Five days after spray}

Buprofezin 25 SC @ $1.25 \mathrm{ml} / \mathrm{l}$ was superior among the various treatments by recording 82.00 per cent mortality of scale insects. The next best treatments were chlorpyriphos $20 \mathrm{EC}$ @ $2 \mathrm{ml} / \mathrm{l}$, acephate $75 \mathrm{SP} @ 1 \mathrm{~g} / \mathrm{l}$, dichlorvos 76 EC@1 ml/l, profenophos 50 EC @ 2 ml/ and lambda-cyhalothrin 5 EC @ $0.5 \mathrm{ml} / \mathrm{l}$. The least mortality of 9.00 per cent was recorded in pongamia oil + tween 80 treatment (Table 7).

Trend was similar when insecticides were applied without FORS. Buprofezin 25 SC @ $1.25 \mathrm{ml} / \mathrm{l}$ was found superior among the various treatments by recording 71.50 per cent mortality and was on par with chlorpyriphos 20 EC @ 2 ml/l, acephate 75 SP @ 1 g/l, profenophos 50 EC @ 2 ml/l, lambdacyhalothrin 5 EC @ $0.5 \mathrm{ml} / 1$ and dichlorvos 76 EC @ $1 \mathrm{ml} / \mathrm{l}$. Least mortality $(13.00 \%)$ was recorded in sunflower oil + tween 80 , cotton oil + tween 80 treatment and sole fish oil rosin soap treatments (Table 8).

\section{Seven days after spray}

Profenophos 50 EC @ $2 \mathrm{ml} / \mathrm{l}$ and acephate 75 SP @ $1 \mathrm{~g} / \mathrm{l}$ were superior among the various treatments by recording 86.00 per cent mortality. The next best treatments were buprofezin 25 SC @1.25 ml/l, chlorpyriphos 20 EC@ 2 ml/1 and dichlorvos 76 EC @ 1 $\mathrm{ml} / \mathrm{l}$. The least per cent mortality (12.00) was recorded in sunflower oil + tween 80 treatment (Table 7).

Trend was similar when insecticides were applied without FORS. 
Table.1 Laboratory evaluation of different insecticides and biorationals with FORS against first instar scale, H. imbricans (Pooled)

\begin{tabular}{|c|c|c|c|c|c|c|}
\hline \multirow[t]{2}{*}{ Tr. No. } & \multirow[t]{2}{*}{ Treatments } & \multirow{2}{*}{$\begin{array}{l}\text { Dosage } \\
(\mathrm{g} / \mathrm{ml} / \mathrm{l})\end{array}$} & \multicolumn{4}{|c|}{ Per cent mortality } \\
\hline & & & 1 DAS & 3 DAS & 5 DAS & 7 DAS \\
\hline $\mathrm{T}_{1}$ & Buprofezin $25 \mathrm{SC}$ & 1.25 & $91.10(72.56) \mathrm{a}$ & $100(90)$ & $100(90)$ & $100(90)$ \\
\hline $\mathrm{T}_{2}$ & Thiamethoxam $25 \mathrm{WG}$ & 0.3 & $81.00(64.16) \mathrm{d}-\mathrm{f}$ & $100(90)$ & $100(90)$ & $100(90)$ \\
\hline $\mathrm{T}_{3}$ & Acetamiprid $20 \mathrm{SP}$ & 0.2 & $83.50(66.05) \mathrm{c}-\mathrm{e}$ & $100(90)$ & $100(90)$ & $100(90)$ \\
\hline $\mathrm{T}_{4}$ & Imidacloprid 17.8 SL & 0.3 & $83.50(66.05) \mathrm{c}-\mathrm{e}$ & $100(90)$ & $100(90)$ & $100(90)$ \\
\hline $\mathrm{T}_{5}$ & Dimethoate $30 \mathrm{EC}$ & 1.7 & $83.50(66.05)$ c-e & $100(90)$ & $100(90)$ & $100(90)$ \\
\hline $\mathrm{T}_{6}$ & Chlorpyriphos $20 \mathrm{EC}$ & 2.0 & $89.50(71.09) \mathrm{a}$ & $100(90)$ & $100(90)$ & $100(90)$ \\
\hline $\mathrm{T}_{7}$ & Acephate 75 SP & 1.0 & $90.01(71.58) \mathrm{a}$ & $100(90)$ & $100(90)$ & $100(90)$ \\
\hline $\mathrm{T}_{8}$ & Deltamethrin $2.8 \mathrm{EC}$ & 0.5 & $81.50(64.52) \mathrm{d}-\mathrm{f}$ & $100(90)$ & $100(90)$ & $100(90)$ \\
\hline $\mathrm{T}_{9}$ & Buprofezin 5.65 SC + Deltamethrin 0.72 EC & 2.5 & $89.51(71.13) \mathrm{a}$ & $100(90)$ & $100(90)$ & $100(90)$ \\
\hline $\mathrm{T}_{10}$ & Lambda-cyhalothrin 5 EC & 0.5 & $89.01(70.64) \mathrm{a}$ & $100(90)$ & $100(90)$ & $100(90)$ \\
\hline $\mathrm{T}_{11}$ & Methomyl 40 SP & 0.3 & $84.52(66.81) \mathrm{cd}$ & $100(90)$ & $100(90)$ & $100(90)$ \\
\hline $\mathrm{T}_{12}$ & Profenophos $50 \mathrm{EC}$ & 2.0 & $88.53(70.18) a b$ & $100(90)$ & $100(90)$ & $100(90)$ \\
\hline $\mathrm{T}_{13}$ & Bifenthrin $10 \mathrm{EC}$ & 0.5 & $86.00(68.03) b c$ & $100(90)$ & $100(90)$ & $100(90)$ \\
\hline $\mathrm{T}_{14}$ & Dichlorvos 76 EC & 1.0 & $90.01(71.58) \mathrm{a}$ & $100(90)$ & $100(90)$ & $100(90)$ \\
\hline $\mathrm{T}_{15}$ & Lecanicillium lecani $\mathrm{WP}+$ tween 80 & $2+0.5$ & $76.51(61.01) \mathrm{g}$ & $100(90)$ & $100(90)$ & $100(90)$ \\
\hline $\mathrm{T}_{16}$ & M. anisopliae $\mathrm{WP}+$ tween 80 & $2+0.5$ & $78.57(62.37) \mathrm{fg}$ & $100(90)$ & $100(90)$ & $100(90)$ \\
\hline $\mathrm{T}_{17}$ & Mealykill & 10.00 & $78.54(62.37) \mathrm{fg}$ & $100(90)$ & $100(90)$ & $100(90)$ \\
\hline $\mathrm{T}_{18}$ & Neemazal 15000 ppm & 5.00 & $70.00(56.78) \mathrm{h}$ & $100(90)$ & $100(90)$ & $100(90)$ \\
\hline $\mathrm{T}_{19}$ & Pongamia oil + tween 80 & $10 \%+0.5$ & $69.51(56.48) \mathrm{h}$ & $100(90)$ & $100(90)$ & $100(90)$ \\
\hline $\mathrm{T}_{20}$ & Cotton seed oil + tween 80 & $10 \%+0.5$ & $70.00(56.78) \mathrm{h}$ & $100(90)$ & $100(90)$ & $100(90)$ \\
\hline $\mathrm{T}_{21}$ & Sunflower oil + tween 80 & $10 \%+0.5$ & $75.51(60.33) \mathrm{g}$ & $100(90)$ & $100(90)$ & $100(90)$ \\
\hline $\mathrm{T}_{22}$ & Fish oil rosin soap & 5.00 & $80.51(63.83)$ ef & $100(90)$ & $100(90)$ & $100(90)$ \\
\hline $\mathrm{T}_{23}$ & Arka suraksha (IIHR Product) & 15.00 & $80.51(63.83)$ ef & $100(90)$ & $100(90)$ & $100(90)$ \\
\hline $\mathrm{T}_{24}$ & Arka rakshak (IIHR Product) & 15.00 & $81.00(64.16) \mathrm{d}-\mathrm{f}$ & $100(90)$ & $100(90)$ & $100(90)$ \\
\hline \multirow[t]{3}{*}{$\mathrm{T}_{25}$} & Untreated control & - & $0.00(0.29)$ & $0.00(0.29)$ & $0.00(0.29)$ & $0.00(0.29)$ \\
\hline & & $\mathbf{S . E m} \pm$ & 1.07 & 0.00 & 0.00 & 0.00 \\
\hline & & $C D(p=0.01)$ & 4.23 & 0.00 & 0.00 & 0.00 \\
\hline
\end{tabular}

DAS - Day after spray

Figures in the parentheses are arc sine transformed values Means followed by same alphabet do not differ significantly by DMRT $(\mathrm{p}=0.05)$ 
Table.2 Laboratory evaluation of different insecticides and biorationals without FORS against first instar scale, H. imbricans (Pooled)

\begin{tabular}{|c|c|c|c|c|c|c|}
\hline \multirow[t]{2}{*}{ Tr. No. } & \multirow[t]{2}{*}{ Treatments } & \multirow{2}{*}{$\begin{array}{l}\text { Dosage } \\
(\mathrm{g} / \mathrm{ml} / \mathrm{l})\end{array}$} & \multicolumn{4}{|c|}{ Per cent mortality } \\
\hline & & & $1 \mathrm{DAS}$ & 3 DAS & 5 DAS & $7 \mathrm{DAS}$ \\
\hline $\mathrm{T}_{1}$ & Buprofezin $25 \mathrm{SC}$ & 1.25 & $90.50(72.10) \mathrm{a}$ & $100(90)$ & $100(90)$ & $100(90)$ \\
\hline $\mathrm{T}_{2}$ & Thiamethoxam $25 \mathrm{WG}$ & 0.3 & $80.50(63.81) b-d$ & $100(90)$ & $100(90)$ & $100(90)$ \\
\hline $\mathrm{T}_{3}$ & Acetamiprid $20 \mathrm{SP}$ & 0.2 & $84.00(66.46) b c$ & $100(90)$ & $100(90)$ & $100(90)$ \\
\hline $\mathrm{T}_{4}$ & Imidacloprid 17.8 SL & 0.3 & $84.50(66.81) b$ & $100(90)$ & $100(90)$ & $100(90)$ \\
\hline $\mathrm{T}_{5}$ & Dimethoate $30 \mathrm{EC}$ & 1.7 & $84.00(66.46) b c$ & $100(90)$ & $100(90)$ & $100(90)$ \\
\hline $\mathrm{T}_{6}$ & Chlorpyriphos $20 \mathrm{EC}$ & 2.0 & $90.50(72.10) \mathrm{a}$ & $100(90)$ & $100(90)$ & $100(90)$ \\
\hline $\mathrm{T}_{7}$ & Acephate $75 \mathrm{SP}$ & 1.0 & $89.50(71.13) \mathrm{a}$ & $100(90)$ & $100(90)$ & $100(90)$ \\
\hline $\mathrm{T}_{8}$ & Deltamethrin $2.8 \mathrm{EC}$ & 0.5 & $81.00(64.15) b-d$ & $100(90)$ & $100(90)$ & $100(90)$ \\
\hline $\mathrm{T}_{9}$ & Buprofezin 5.65 SC + Deltamethrin 0.72 EC & 2.5 & $80.00(63.44) b-d$ & $100(90)$ & $100(90)$ & $100(90)$ \\
\hline $\mathrm{T}_{10}$ & Lambda-cyhalothrin 5 EC & 0.5 & $90.50(72.05) \mathrm{a}$ & $100(90)$ & $100(90)$ & $100(90)$ \\
\hline $\mathrm{T}_{11}$ & Methomyl 40 SP & 0.3 & $84.50(66.81) b$ & $100(90)$ & $100(90)$ & $100(90)$ \\
\hline $\mathrm{T}_{12}$ & Profenophos $50 \mathrm{EC}$ & 2.0 & $90.50(72.10) \mathrm{a}$ & $100(90)$ & $100(90)$ & $100(90)$ \\
\hline $\mathrm{T}_{13}$ & Bifenthrin $10 \mathrm{EC}$ & 0.5 & $80.50(63.81) b-d$ & $100(90)$ & $100(90)$ & $100(90)$ \\
\hline $\mathrm{T}_{14}$ & Dichlorvos 76 EC & 1.0 & $90.00(71.58) \mathrm{a}$ & $100(90)$ & $100(90)$ & $100(90)$ \\
\hline $\mathrm{T}_{15}$ & Lecanicillium lecani $\mathrm{WP}+$ tween 80 & $2+0.5$ & $77.50(61.68) \mathrm{d}$ & $100(90)$ & $100(90)$ & $100(90)$ \\
\hline $\mathrm{T}_{16}$ & M. anisopliae WP + tween 80 & $2+0.5$ & $79.00(62.72) \mathrm{d}$ & $100(90)$ & $100(90)$ & $100(90)$ \\
\hline $\mathrm{T}_{17}$ & Mealykill & 10.00 & $78.50(62.37) \mathrm{d}$ & $100(90)$ & $100(90)$ & $100(90)$ \\
\hline $\mathrm{T}_{18}$ & Neemazal 15000 ppm & 5.00 & $80.00(63.44) b-d$ & $100(90)$ & $100(90)$ & $100(90)$ \\
\hline $\mathrm{T}_{19}$ & Pongamia oil + tween 80 & $10 \%+0.5$ & $79.50(63.08) \mathrm{cd}$ & $100(90)$ & $100(90)$ & $100(90)$ \\
\hline $\mathrm{T}_{20}$ & Cotton seed oil + tween 80 & $10 \%+0.5$ & $70.00(56.78) \mathrm{e}$ & $100(90)$ & $100(90)$ & $100(90)$ \\
\hline $\mathrm{T}_{21}$ & Sunflower oil + tween 80 & $10 \%+0.5$ & $72.50(58.37) \mathrm{e}$ & $100(90)$ & $100(90)$ & $100(90)$ \\
\hline $\mathrm{T}_{22}$ & Fish oil rosin soap & 5.00 & $70.00(56.78) \mathrm{e}$ & $100(90)$ & $100(90)$ & $100(90)$ \\
\hline $\mathrm{T}_{23}$ & Arka suraksha (IIHR Product) & 15.00 & $80.50(63.81) b-d$ & $100(90)$ & $100(90)$ & $100(90)$ \\
\hline $\mathrm{T}_{24}$ & Arka rakshak (IIHR Product) & 15.00 & $81.00(64.16) b-d$ & $100(90)$ & $100(90)$ & $100(90)$ \\
\hline \multirow[t]{3}{*}{$\mathrm{T}_{25}$} & Untreated control & - & $0.00(0.29)$ & $0.00(0.29)$ & $0.00(0.29)$ & $0.00(0.29)$ \\
\hline & & S.Em \pm & 1.26 & 0.00 & 0.00 & 0.00 \\
\hline & & $\mathrm{CD}(\mathrm{p}=0.01)$ & 4.96 & 0.00 & 0.00 & 0.00 \\
\hline
\end{tabular}

DAS - Day after spray $\quad$ Figures in the parentheses are arc sine transformed values

Means followed by same alphabet do not differ significantly by DMRT $(\mathrm{p}=0.05)$ 
Table.3 Laboratory evaluation of different insecticides and biorationals with FORS against second instar scale, H. imbricans (Pooled)

\begin{tabular}{|c|c|c|c|c|c|c|}
\hline \multirow{2}{*}{$\begin{array}{l}\text { Tr. } \\
\text { No. }\end{array}$} & \multirow[t]{2}{*}{ Treatments } & \multirow{2}{*}{$\begin{array}{l}\text { Dosage } \\
(\mathrm{g} / \mathrm{ml} / \mathrm{l})\end{array}$} & \multicolumn{4}{|c|}{ Per cent mortality } \\
\hline & & & $1 \mathrm{DAS}$ & $3 \mathrm{DAS}$ & 5 DAS & $7 \mathrm{DAS}$ \\
\hline $\mathrm{T}_{1}$ & Buprofezin $25 \mathrm{SC}$ & 1.25 & $56.51(48.73) \mathrm{a}$ & $64.51(53.53) \mathrm{a}$ & $81.00(64.15) b$ & $99.00(84.26) \mathrm{a}$ \\
\hline $\mathrm{T}_{2}$ & Thiamethoxam25 WG & 0.3 & $35.00(36.27) \mathrm{de}$ & $40.00(39.20) \mathrm{d}-\mathrm{g}$ & $49.50(44.71) \mathrm{c}$ & $51.00(45.57) \mathrm{c}-\mathrm{e}$ \\
\hline $\mathrm{T}_{3}$ & Acetamiprid $20 \mathrm{SP}$ & 0.2 & $35.00(36.26) \mathrm{de}$ & $41.51(40.08) \mathrm{d}-\mathrm{f}$ & $49.00(44.42) \mathrm{c}$ & $51.50(45.85) \mathrm{c}-\mathrm{e}$ \\
\hline $\mathrm{T}_{4}$ & Imidacloprid 17.8 SL & 0.3 & $41.50(40.10) b c$ & 46.00 (42.69) b-e & $54.00(47.29) \mathrm{c}$ & $57.50(49.31) \mathrm{c}$ \\
\hline $\mathrm{T}_{5}$ & Dimethoate $30 \mathrm{EC}$ & 1.7 & $37.51(37.76) c-e$ & $43.00(40.94) \mathrm{c}-\mathrm{e}$ & $50.50(45.28) \mathrm{c}$ & $53.00(46.72) \mathrm{c}-\mathrm{e}$ \\
\hline $\mathrm{T}_{6}$ & Chlorpyriphos $20 \mathrm{EC}$ & 2.0 & $41.51(40.58) \mathrm{b}$ & $57.10(49.04) \mathrm{a}-\mathrm{d}$ & $85.00(67.31) \mathrm{ab}$ & $96.50(79.24) \mathrm{b}$ \\
\hline $\mathrm{T}_{7}$ & Acephate 75 SP & 1.0 & $41.51(40.58) b$ & $60.00(50.92) \mathrm{a}-\mathrm{c}$ & $90.00(71.56) \mathrm{a}$ & $94.00(75.82) \mathrm{b}$ \\
\hline $\mathrm{T}_{8}$ & Deltamethrin $2.8 \mathrm{EC}$ & 0.5 & $33.51(35.36) \mathrm{e}$ & $37.00(37.42) \mathrm{e}-\mathrm{h}$ & $47.00(43.27) \mathrm{c}$ & $50.00(45.00) \mathrm{de}$ \\
\hline $\mathrm{T}_{9}$ & Buprofezin 5.65 SC + Deltamethrin 0.72 EC & 2.5 & $40.00(39.23) \mathrm{cd}$ & $45.00(42.10) \mathrm{b}-\mathrm{e}$ & $54.51(47.58) \mathrm{c}$ & $55.50(48.15) \mathrm{cd}$ \\
\hline $\mathrm{T}_{10}$ & Lambda-cyhalothrin $5 \mathrm{EC}$ & 0.5 & $43.00(40.94) \mathrm{b}$ & $61.00(51.49) \mathrm{ab}$ & $91.00(72.56) \mathrm{a}$ & $94.50(76.56) \mathrm{b}$ \\
\hline $\mathrm{T}_{11}$ & Methomyl 40 SP & 0.3 & $35.01(36.25) \mathrm{de}$ & $37.00(37.42)$ e-h & $46.10(42.70) \mathrm{c}$ & $48.00(43.85) \mathrm{e}$ \\
\hline $\mathrm{T}_{12}$ & Profenophos $50 \mathrm{EC}$ & 2.0 & $36.51(37.15) \mathrm{c}-\mathrm{e}$ & $39.50(38.89) \mathrm{d}-\mathrm{g}$ & $48.12(43.85) \mathrm{c}$ & $50.00(45.00) \mathrm{de}$ \\
\hline $\mathrm{T}_{13}$ & Bifenthrin $10 \mathrm{EC}$ & 0.5 & $37.12(37.46) \mathrm{c}-\mathrm{e}$ & $39.51(38.93) \mathrm{d}-\mathrm{g}$ & $48.00(43.85) \mathrm{c}$ & $49.50(44.71) \mathrm{de}$ \\
\hline $\mathrm{T}_{14}$ & Dichlorvos $76 \mathrm{EC}$ & 1.0 & $43.00(40.94) \mathrm{b}$ & $60.54(51.15) a b$ & $88.12(69.73) a b$ & $94.50(76.56) \mathrm{b}$ \\
\hline $\mathrm{T}_{15}$ & Lecanicillium lecani $\mathrm{WP}+$ tween 80 & $2+0.5$ & $11.51(19.83) \mathrm{i}$ & $15.00(22.78) \mathrm{i}$ & $16.51(23.97) \mathrm{de}$ & $19.00(25.84) \mathrm{ij}$ \\
\hline $\mathrm{T}_{16}$ & M. anisopliae WP + tween 80 & $2+0.5$ & $16.00(23.57) \mathrm{gh}$ & $18.55(25.51) \mathrm{i}$ & $21.50(27.62) \mathrm{e}$ & $22.50(30.32) \mathrm{f}-\mathrm{h}$ \\
\hline $\mathrm{T}_{17}$ & Mealykill & 10.00 & $19.53(26.22) \mathrm{fg}$ & $21.00(27.27) \mathrm{hi}$ & $26.00(30.65) \mathrm{e}$ & $28.50(32.26) \mathrm{fg}$ \\
\hline $\mathrm{T}_{18}$ & Neemazal 15000 ppm & 5.00 & $18.14(25.20) \mathrm{f}-\mathrm{h}$ & $21.00(27.27) \mathrm{hi}$ & $22.00(27.97) \mathrm{de}$ & $24.00(29.33) \mathrm{f}-\mathrm{i}$ \\
\hline $\mathrm{T}_{19}$ & Pongamia oil + tween 80 & $10 \%+0.5$ & $14.52(22.39) \mathrm{hi}$ & $15.00(22.78) \mathrm{i}$ & $16.50(23.96) \mathrm{e}$ & $17.00(24.35) \mathrm{j}$ \\
\hline $\mathrm{T}_{20}$ & Cotton seed oil + tween 80 & $10 \%+0.5$ & $16.00(23.57) \mathrm{gh}$ & $17.51(24.73) \mathrm{i}$ & $10.00(18.40) \mathrm{e}$ & $20.50(26.92) h-j$ \\
\hline $\mathrm{T}_{21}$ & Sunflower oil + tween 80 & $10 \%+0.5$ & $16.00(23.57) \mathrm{gh}$ & $17.51(24.73) \mathrm{i}$ & $19.50(26.20) \mathrm{de}$ & $22.50(28.31) \mathrm{g}-\mathrm{j}$ \\
\hline $\mathrm{T}_{22}$ & Fish oil rosin soap & 5.00 & 18.55 (25.51) fgh & $21.50(27.62) \mathrm{hi}$ & $29.00(32.58) \mathrm{d}$ & $30.00(33.21) \mathrm{f}$ \\
\hline $\mathrm{T}_{23}$ & Arka suraksha (IIHR Product) & 15.00 & $22.52(28.33) \mathrm{f}$ & $25.51(30.33) f-i$ & $26.00(30.65) \mathrm{de}$ & $28.50(32.36) \mathrm{fg}$ \\
\hline $\mathrm{T}_{24}$ & Arka rakshak (IIHR Product) & 15.00 & $20.52(26.93) \mathrm{f}$ & $23.52(29.01) \mathrm{g}-\mathrm{i}$ & $26.50(30.32) \mathrm{de}$ & $27.00(31.30) \mathrm{fg}$ \\
\hline \multirow[t]{3}{*}{$\mathrm{T}_{25}$} & Untreated control & - & $0.00(0.29)$ & $0.00(0.29)$ & $0.00(0.29)$ & $0.00(0.29)$ \\
\hline & & S.Em \pm & 1.57 & 2.13 & 2.04 & 1.73 \\
\hline & & $\mathrm{CD}(\mathrm{p}=0.01)$ & 6.18 & 6.41 & 6.05 & 6.84 \\
\hline
\end{tabular}

DAS - Day after spray

Figures in the parentheses are arc sine transformed values

Means followed by same alphabet do not differ significantly by DMRT $(\mathrm{p}=0.05)$ 
Table.4 Laboratory evaluation of different insecticides and biorationals without FORS second instar scale, H. imbricans (Pooled)

\begin{tabular}{|c|c|c|c|c|c|c|}
\hline \multirow[t]{2}{*}{ Tr. No. } & \multirow[t]{2}{*}{ Treatments } & \multirow{2}{*}{$\begin{array}{l}\text { Dosage } \\
(\mathrm{g} / \mathrm{ml} / \mathrm{l})\end{array}$} & \multicolumn{4}{|c|}{ Per cent mortality } \\
\hline & & & 1 DAS & 3 DAS & 5 DAS & 7 DAS \\
\hline $\mathbf{T}_{1}$ & Buprofezin 25 SC & 1.25 & $51.50(45.85) \mathrm{a}$ & $66.00(54.34) \mathrm{a}$ & $86.50(68.44) \mathrm{a}$ & $95.50(78.91) \mathrm{a}$ \\
\hline $\mathbf{T}_{2}$ & Thiamethoxam $25 \mathrm{WG}$ & 0.3 & $32.50(34.75) \mathrm{e}$ & $40.50(39.52) \mathrm{cd}$ & $46.50(42.99) \mathrm{c}-\mathrm{e}$ & $48.00(43.85) \mathrm{c}$ \\
\hline $\mathbf{T}_{3}$ & Acetamiprid $20 \mathrm{SP}$ & 0.2 & $32.00(34.43) \mathrm{e}$ & $41.50(40.09) \mathrm{cd}$ & $45.00(42.12) \mathrm{c}-\mathrm{e}$ & $49.50(44.71) \mathrm{c}$ \\
\hline $\mathbf{T}_{4}$ & Imidacloprid $17.8 \mathrm{SL}$ & 0.3 & $39.00(38.64) \mathrm{c}$ & $44.50(41.82) b c$ & $51.50(45.86) \mathrm{c}$ & $53.50(47.01) \mathrm{c}$ \\
\hline $\mathbf{T}_{5}$ & Dimethoate $30 \mathrm{EC}$ & 1.7 & $35.50(36.57) \mathrm{c}-\mathrm{e}$ & $42.50(40.66) b-d$ & $48.00(43.85) \mathrm{c}-\mathrm{e}$ & $52.00(46.14) \mathrm{c}$ \\
\hline $\mathbf{T}_{6}$ & Chlorpyriphos $20 \mathrm{EC}$ & 2.0 & $41.50(40.09) \mathrm{b}$ & $55.00(47.87) \mathrm{ab}$ & $78.50(62.44) \mathrm{b}$ & $95.00(77.14) \mathrm{ab}$ \\
\hline $\mathbf{T}_{7}$ & Acephate 75 SP & 1.0 & $41.50(40.09) \mathrm{b}$ & $58.00(49.68) \mathrm{a}$ & $80.50(63.81) b$ & $91.00(72.56) \mathrm{b}$ \\
\hline $\mathbf{T}_{8}$ & Deltamethrin 2.8 EC & 0.5 & $31.50(34.13) \mathrm{e}$ & $36.00(36.84) \mathrm{cd}$ & $42.00(40.39) \mathrm{e}$ & $47.50(43.56) \mathrm{c}$ \\
\hline $\mathbf{T}_{9}$ & Buprofezin 5.65 SC + Deltamethrin 0.72 EC & 2.5 & $38.00(38.05) \mathrm{c}$ & $43.50(41.24) \mathrm{bc}$ & $50.00(45.00) \mathrm{cd}$ & $53.00(46.72) \mathrm{c}$ \\
\hline $\mathbf{T}_{10}$ & Lambda-cyhalothrin 5 EC & 0.5 & $42.50(40.66) \mathrm{b}$ & $59.50(50.56) \mathrm{a}$ & $80.50(63.81) b$ & $92.50(74.11) \mathrm{ab}$ \\
\hline $\mathbf{T}_{11}$ & Methomyl 40 SP & 0.3 & $33.00(35.04) \mathrm{de}$ & $36.00(36.84) \mathrm{cd}$ & $41.50(40.09) \mathrm{e}$ & $46.50(42.99) \mathrm{c}$ \\
\hline $\mathbf{T}_{12}$ & Profenophos $50 \mathrm{EC}$ & 2.0 & $35.00(36.26) \mathrm{c}-\mathrm{e}$ & $39.00(38.61) \mathrm{cd}$ & $43.50(41.26) \mathrm{de}$ & $49.00(44.42) \mathrm{c}$ \\
\hline $\mathbf{T}_{13}$ & Bifenthrin $10 \mathrm{EC}$ & 0.5 & $36.50(37.16) \mathrm{cd}$ & $38.50(38.34) \mathrm{cd}$ & $44.00(41.55) \mathrm{de}$ & $50.50(45.28) \mathrm{c}$ \\
\hline $\mathbf{T}_{14}$ & Dichlorvos 76 EC & 1.0 & $48.50(44.14) \mathrm{ab}$ & $58.50(49.89) \mathrm{a}$ & $80.50(63.81) \mathrm{b}$ & $93.50(75.23) \mathrm{ab}$ \\
\hline $\mathbf{T}_{15}$ & Lecanicillium lecani $\mathrm{WP}+$ tween 80 & $2+0.5$ & $18.00(25.10) \mathrm{gh}$ & $22.00(27.97)$ ef & $25.50(28.93) \mathrm{g}-\mathrm{i}$ & $27.00(31.30) \mathrm{df}$ \\
\hline $\mathbf{T}_{16}$ & M. anisopliae $\mathrm{WP}+$ tween 80 & $2+0.5$ & $15.00(22.78) \mathrm{ij}$ & $19.50(26.20)$ ef & $22.50(28.39) \mathrm{g}-\mathrm{i}$ & $24.00(29.33) \mathrm{e}-\mathrm{g}$ \\
\hline $\mathbf{T}_{17}$ & Mealykill & 10.00 & $19.00(25.84) \mathrm{g}$ & $20.00(26.56)$ ef & $23.50(28.99) \mathrm{g}-\mathrm{i}$ & $28.00(31.94) \mathrm{de}$ \\
\hline $\mathbf{T}_{18}$ & Neemazal 15000 ppm & 5.00 & $27.00(31.30) \mathrm{f}$ & $30.00(33.21) \mathrm{de}$ & $31.50(34.14) \mathrm{f}$ & $32.50(34.75) d$ \\
\hline $\mathbf{T}_{19}$ & Pongamia oil + tween 80 & $10 \%+0.5$ & $12.50(20.70) \mathrm{j}-1$ & $14.00(21.97) \mathrm{f}$ & $16.00(23.58) \mathrm{jk}$ & $19.00(25.84) \mathrm{gh}$ \\
\hline $\mathbf{T}_{20}$ & Cotton seed oil + tween 80 & $10 \%+0.5$ & $14.00(21.97) \mathrm{i}-\mathrm{k}$ & $15.50(23.18) \mathrm{f}$ & $18.50(25.47) h-j$ & $19.50(26.26) \mathrm{f}-\mathrm{h}$ \\
\hline $\mathbf{T}_{21}$ & Sunflower oil + tween 80 & $10 \%+0.5$ & $10.50(18.90) 1$ & $12.00(20.26) \mathrm{f}$ & $13.00(21.13) \mathrm{k}$ & $14.00(21.97) \mathrm{h}$ \\
\hline $\mathbf{T}_{22}$ & Fish oil rosin soap & 5.00 & $12.00(20.26) \mathrm{kl}$ & $15.50(22.78) \mathrm{f}$ & $18.00(25.10) \mathrm{ij}$ & $22.50(28.31) \mathrm{e}-\mathrm{g}$ \\
\hline $\mathbf{T}_{23}$ & Arka suraksha (IIHR Product) & 15.00 & $15.50(23.18)$ hi & $18.50(25.47) \mathrm{f}$ & $24.00(29.33) \mathrm{gh}$ & $26.00(30.65) d-g$ \\
\hline $\mathbf{T}_{24}$ & Arka rakshak (IIHR Product) & 15.00 & $19.50(26.20) \mathrm{g}$ & $22.00(27.97)$ ef & $27.00(31.30) \mathrm{fg}$ & $29.00(32.58) \mathrm{de}$ \\
\hline \multirow[t]{3}{*}{$\mathbf{T}_{25}$} & Untreated control & - & $0.00(0.29)$ & $0.00(0.29)$ & $0.00(0.29)$ & $0.00(0.29)$ \\
\hline & & S.Em \pm & 1.19 & 2.90 & 1.98 & 2.04 \\
\hline & & CD $(p=0.01)$ & 4.68 & 4.37 & 4.39 & 4.03 \\
\hline
\end{tabular}

DAS - Day after spray

Figures in the parentheses are arc sine transformed values

Means followed by same alphabet do not differ significantly by DMRT $(p=0.05)$ 
Table.5 Laboratory evaluation of different insecticides and biorationals with FORS against third instar scale, H. imbricans (Pooled)

\begin{tabular}{|c|c|c|c|c|c|c|}
\hline \multirow[t]{2}{*}{ Tr. No. } & \multirow[t]{2}{*}{ Treatments } & \multirow{2}{*}{$\begin{array}{l}\text { Dosage } \\
(\mathrm{g} / \mathrm{ml} / \mathrm{l})\end{array}$} & \multicolumn{4}{|c|}{ Per cent mortality } \\
\hline & & & 1 DAS & 3 DAS & 5 DAS & 7 DAS \\
\hline$T_{1}$ & Buprofezin $25 \mathrm{SC}$ & 1.25 & $44.50(41.84) \mathrm{a}$ & $59.50(50.47) \mathrm{b}$ & $82.50(65.27) a b$ & $92.50(74.10) \mathrm{a}$ \\
\hline $\mathbf{T}_{2}$ & Thiamethoxam $25 \mathrm{WG}$ & 0.3 & $25.00(30.00) \mathrm{c}$ & $31.50(34.14) d$ & $35.50(36.57) \mathrm{e}$ & $43.00(40.97) \mathrm{fg}$ \\
\hline $\mathbf{T}_{3}$ & Acetamiprid 20 SP & 0.2 & $25.00(30.00) \mathrm{c}$ & $31.50(34.14) \mathrm{d}$ & $35.00(36.27) \mathrm{e}$ & $40.50(39.52) \mathrm{gh}$ \\
\hline $\mathbf{T}_{4}$ & Imidacloprid 17.8 SL & 0.3 & $25.00(30.00) \mathrm{c}$ & $33.50(35.36) d$ & $37.50(37.75) \mathrm{e}$ & $43.00(40.97) \mathrm{fg}$ \\
\hline $\mathbf{T}_{5}$ & Dimethoate $30 \mathrm{EC}$ & 1.7 & $30.00(33.19) b c$ & $35.50(36.57) \mathrm{d}$ & $39.50(38.93) \mathrm{e}$ & $43.00(40.97) \mathrm{fg}$ \\
\hline $\mathbf{T}_{6}$ & Chlorpyriphos $20 \mathrm{EC}$ & 2.0 & $47.00(43.27) \mathrm{a}$ & $59.50(50.47) b$ & $81.00(64.15) b$ & $85.50(67.61) \mathrm{d}$ \\
\hline $\mathbf{T}_{7}$ & Acephate 75 SP & 1.0 & $50.50(45.28) \mathrm{a}$ & $59.50(50.47) \mathrm{b}$ & $81.00(64.15) b$ & $88.00(69.73) b c$ \\
\hline $\mathbf{T}_{8}$ & Deltamethrin $2.8 \mathrm{EC}$ & 0.5 & $28.50(32.26) b c$ & $35.00(36.27) \mathrm{d}$ & $39.50(38.93) \mathrm{e}$ & $44.50(41.84) \mathrm{f}$ \\
\hline $\mathbf{T}_{9}$ & Buprofezin 5.65 SC + Deltamethrin 0.72 EC & 2.5 & $34.00(35.65) \mathrm{b}$ & $41.50(40.10) \mathrm{c}$ & $45.00(42.13) \mathrm{cd}$ & $47.50(43.56) \mathrm{e}$ \\
\hline $\mathbf{T}_{10}$ & Lambda-cyhalothrin $5 \mathrm{EC}$ & 0.5 & $50.00(45.00) \mathrm{a}$ & $75.00(60.07) \mathrm{ab}$ & $86.00(68.02) \mathrm{a}$ & $89.00(70.63) b$ \\
\hline $\mathbf{T}_{11}$ & Methomyl 40 SP & 0.3 & $25.50(30.32) \mathrm{c}$ & $31.50(34.14) \mathrm{dc}$ & $36.00(36.86) \mathrm{e}$ & $43.00(40.97) \mathrm{fg}$ \\
\hline $\mathbf{T}_{12}$ & Profenophos $50 \mathrm{EC}$ & 2.0 & $34.00(35.65) b$ & $44.00(41.55) \mathrm{c}$ & $47.00(43.27) \mathrm{c}$ & $50.00(45.00) \mathrm{e}$ \\
\hline $\mathbf{T}_{13}$ & Bifenthrin $10 \mathrm{EC}$ & 0.5 & $30.00(33.19) b c$ & $34.00(35.65) \mathrm{d}$ & $40.00(39.23) \mathrm{de}$ & $43.50(41.26) \mathrm{fg}$ \\
\hline $\mathbf{T}_{14}$ & Dichlorvos $76 \mathrm{EC}$ & 1.0 & $50.00(45.00) \mathrm{a}$ & $77.50(61.69) \mathrm{a}$ & $81.00(64.15) b$ & $86.00(68.02) \mathrm{cd}$ \\
\hline $\mathbf{T}_{15}$ & Lecanicillium lecani $\mathrm{WP}+$ tween 80 & $2+0.5$ & $10.50(18.90) \mathrm{e}$ & $12.50(20.70) \mathrm{f}-\mathrm{h}$ & $13.50(21.55)$ hi & $15.50(23.18) \mathrm{kl}$ \\
\hline $\mathbf{T}_{16}$ & M. anisopliae $\mathrm{WP}+$ tween 80 & $2+0.5$ & $10.00(18.43) \mathrm{e}$ & $14.50(22.38) \mathrm{e}-\mathrm{g}$ & $15.50(23.18) \mathrm{f}-\mathrm{h}$ & $16.00(23.57) \mathrm{kl}$ \\
\hline $\mathbf{T}_{17}$ & Mealykill & 10.00 & $10.00(18.43) \mathrm{e}$ & $15.50(23.18)$ ef & $16.00(23.57) \mathrm{f}-\mathrm{h}$ & $17.50(24.72) \mathrm{k}$ \\
\hline $\mathbf{T}_{18}$ & Neemazal 15000 ppm & 5.00 & $15.50(23.18) \mathrm{d}$ & $17.50(24.72) \mathrm{e}$ & $18.00(25.10) \mathrm{fg}$ & $20.50(26.92) \mathrm{i}$ \\
\hline $\mathbf{T}_{19}$ & Pongamia oil + tween 80 & $10 \%+0.5$ & $10.00(18.43) \mathrm{e}$ & $10.50(18.90)$ hi & $11.50(19.82) \mathrm{i}$ & $12.50(20.70) \mathrm{m}$ \\
\hline $\mathbf{T}_{20}$ & Cotton seed oil + tween 80 & $10 \%+0.5$ & $9.00(17.45) \mathrm{e}$ & $10.50(18.90) \mathrm{i}$ & $11.00(19.36) \mathrm{i}$ & $12.50(20.70) \mathrm{m}$ \\
\hline $\mathbf{T}_{21}$ & Sunflower oil + tween 80 & $10 \%+0.5$ & $8.00(16.42) \mathrm{e}$ & $9.00(17.45) \mathrm{i}$ & $10.50(18.90) \mathrm{i}$ & $11.00(19.36) \mathrm{m}$ \\
\hline $\mathbf{T}_{22}$ & Fish oil rosin soap & 5.00 & $11.50(19.82) \mathrm{de}$ & $12.00(20.26) \mathrm{gh}$ & $14.00(21.97) \mathrm{g}-\mathrm{i}$ & $15.00(22.78) 1$ \\
\hline $\mathbf{T}_{23}$ & Arka suraksha (IIHR Product) & 15.00 & $10.00(18.43) \mathrm{e}$ & $15.00(22.78) \mathrm{e}-\mathrm{g}$ & $18.00(25.10) \mathrm{fg}$ & $23.00(28.65) \mathrm{i}$ \\
\hline $\mathbf{T}_{24}$ & Arka rakshak (IIHR Product) & 15.00 & $10.50(18.90) \mathrm{e}$ & $16.50(23.96) \mathrm{e}$ & $19.50(26.20) \mathrm{f}$ & $25.50(30.32) \mathrm{i}$ \\
\hline \multirow[t]{3}{*}{$\mathbf{T}_{25}$} & Untreated control & - & $0.00(0.29)$ & $0.00(0.29)$ & $0.00(0.29)$ & $0.00(0.29)$ \\
\hline & & S.Em \pm & 1.73 & 1.30 & 0.95 & 0.99 \\
\hline & & $\operatorname{CD}(p=0.01)$ & 5.60 & 5.11 & 3.76 & 3.92 \\
\hline
\end{tabular}

DAS - Day after spray

Figures in the parentheses are arc sine transformed values

Means followed by same alphabet do not differ significantly by DMRT $(p=0.05)$ 
Table.6 Laboratory evaluation of different insecticides and biorationals without FORS against third instar scale, $H$. imbricans (Pooled)

\begin{tabular}{|c|c|c|c|c|c|c|}
\hline \multirow[t]{2}{*}{ Tr. No. } & \multirow[t]{2}{*}{ Treatments } & \multirow{2}{*}{$\begin{array}{l}\text { Dosage } \\
(\mathrm{g} / \mathrm{ml} / \mathrm{l})\end{array}$} & \multicolumn{4}{|c|}{ Per cent mortality } \\
\hline & & & 1 DAS & 3 DAS & 5 DAS & 7 DAS \\
\hline $\mathbf{T}_{1}$ & Buprofezin 25 SC & 1.25 & $43.00(40.97) \mathrm{a}$ & $59.50(50.47) \mathrm{d}$ & $79.00(62.72) \mathrm{a}$ & $88.50(70.18)$ a \\
\hline $\mathbf{T}_{2}$ & Thiamethoxam25 WG & 0.3 & $23.50(28.98) \mathrm{c}-\mathrm{f}$ & $30.50(33.51) \mathrm{fg}$ & $34.50(35.96)$ ef & $35.50(36.57) \mathrm{eh}$ \\
\hline $\mathbf{T}_{3}$ & Acetamiprid $20 \mathrm{SP}$ & 0.2 & $25.50(30.30) \mathrm{c}-\mathrm{e}$ & $30.00(33.20) \mathrm{g}$ & $33.50(35.36) \mathrm{f}$ & $39.50(38.93) \mathrm{fg}$ \\
\hline $\mathbf{T}_{4}$ & Imidacloprid 17.8 SL & 0.3 & $25.50(30.32) \mathrm{c}-\mathrm{e}$ & $34.00(35.66) \mathrm{fg}$ & $36.00(36.86)$ ef & $42.50(40.68)$ ef \\
\hline $\mathbf{T}_{5}$ & Dimethoate $30 \mathrm{EC}$ & 1.7 & $29.50(32.87) b c$ & $34.50(35.96) \mathrm{f}$ & $38.00(38.05)$ ef & $42.00(40.39)$ ef \\
\hline $\mathbf{T}_{6}$ & Chlorpyriphos $20 \mathrm{EC}$ & 2.0 & $45.00(42.12) \mathrm{a}$ & $64.50(53.42) b c$ & $64.50(53.42) b$ & $86.50(68.44) a b$ \\
\hline $\mathbf{T}_{7}$ & Acephate 75 SP & 1.0 & $47.50(43.56) \mathrm{a}$ & $63.00(52.53) \mathrm{cd}$ & $64.50(53.42) \mathrm{b}$ & $73.00(58.69) \mathrm{c}$ \\
\hline $\mathbf{T}_{8}$ & Deltamethrin $2.8 \mathrm{EC}$ & 0.5 & $28.00(31.94) b-d$ & $32.50(34.75) \mathrm{fg}$ & $37.50(37.75)$ ef & $42.00(40.39)$ ef \\
\hline $\mathbf{T}_{9}$ & Buprofezin 5.65 SC + Deltamethrin 0.72 EC & 2.5 & $33.50(35.31) \mathrm{b}$ & $39.00(38.64) \mathrm{e}$ & $43.50(41.26) \mathrm{cd}$ & $46.00(42.70) \mathrm{de}$ \\
\hline $\mathbf{T}_{10}$ & Lambda-cyhalothrin 5 EC & 0.5 & $48.00(43.85) \mathrm{a}$ & $67.50(55.24) \mathrm{ab}$ & $70.00(56.79) b$ & $86.00(68.03) \mathrm{ab}$ \\
\hline $\mathbf{T}_{11}$ & Methomyl 40 SP & 0.3 & $24.50(29.66) \mathrm{c}-\mathrm{f}$ & $31.00(33.83) \mathrm{fg}$ & $35.50(36.57)$ ef & $40.00(39.23) \mathrm{f}$ \\
\hline $\mathbf{T}_{12}$ & Profenophos $50 \mathrm{EC}$ & 2.0 & $33.00(35.04) \mathrm{b}$ & $40.50(39.52) \mathrm{e}$ & $45.50(42.41) \mathrm{c}$ & $49.00(44.42) \mathrm{d}$ \\
\hline $\mathbf{T}_{13}$ & Bifenthrin $10 \mathrm{EC}$ & 0.5 & $29.50(32.87) b c$ & $33.00(35.04) \mathrm{fg}$ & $39.50(38.93) \mathrm{de}$ & $42.50(40.68)$ ef \\
\hline $\mathbf{T}_{14}$ & Dichlorvos $76 \mathrm{EC}$ & 1.0 & $48.00(43.85) \mathrm{a}$ & $70.50(57.10) \mathrm{a}$ & $78.50(62.37) \mathrm{a}$ & $81.00(64.15) b$ \\
\hline $\mathbf{T}_{15}$ & Lecanicillium lecani $\mathrm{WP}+$ tween 80 & $2+0.5$ & $20.00(26.56)$ e-g & $20.50(26.92) \mathrm{i}$ & $22.00(27.97) \mathrm{hi}$ & $24.50(29.66) \mathrm{jk}$ \\
\hline $\mathbf{T}_{16}$ & Manisopliae WP + tween 80 & $2+0.5$ & $18.50(25.47) \mathrm{f}-\mathrm{h}$ & $24.00(29.33) \mathrm{h}$ & $25.50(30.32) \mathrm{gh}$ & $27.00(31.30) \mathrm{j}$ \\
\hline $\mathbf{T}_{17}$ & Mealykill & 10.00 & $17.00(24.35) \mathrm{g}-\mathrm{i}$ & $19.50(26.20) \mathrm{i}$ & $24.50(29.66) \mathrm{gh}$ & $26.00(30.65) \mathrm{j}$ \\
\hline $\mathbf{T}_{18}$ & Neemazal 15000 ppm & 5.00 & $22.00(27.97) \mathrm{d}-\mathrm{g}$ & $25.00(30.00) \mathrm{h}$ & $28.50(32.26) \mathrm{g}$ & $31.50(34.14) \mathrm{hi}$ \\
\hline $\mathbf{T}_{19}$ & Pongamia oil + tween 80 & $10 \%+0.5$ & $10.50(18.90) \mathrm{j}$ & $12.00(20.26) \mathrm{j}$ & $14.50(21.97) \mathrm{k}$ & $15.00(22.79) 1$ \\
\hline $\mathbf{T}_{20}$ & Cotton seed oil + tween 80 & $10 \%+0.5$ & $12.50(20.67) \mathrm{ij}$ & $13.50(21.55) \mathrm{j}$ & $15.00(22.79) \mathrm{k}$ & $17.50(24.72) 1$ \\
\hline $\mathbf{T}_{21}$ & Sunflower oil + tween 80 & $10 \%+0.5$ & $10.00(18.34) \mathrm{j}$ & $12.50(20.67) \mathrm{j}$ & $13.50(21.13) \mathrm{k}$ & $14.50(22.38) 1$ \\
\hline $\mathbf{T}_{22}$ & Fish oil rosin soap & 5.00 & $11.00(19.20) \mathrm{j}$ & $13.00(21.12) \mathrm{j}$ & $16.00(23.57) \mathrm{jk}$ & $17.50(24.72) 1$ \\
\hline $\mathbf{T}_{23}$ & Arka suraksha (IIHR Product) & 15.00 & $14.50(22.30) h-j$ & $18.50(25.47) \mathrm{i}$ & $19.50(26.20) \mathrm{ij}$ & $22.00(27.97) \mathrm{k}$ \\
\hline $\mathbf{T}_{24}$ & Arka rakshak (IIHR Product) & 15.00 & $14.50(22.30) h-j$ & $19.50(26.20) \mathrm{I}$ & $23.00(28.65) \mathrm{hi}$ & $25.50(32.26) \mathrm{ij}$ \\
\hline \multirow[t]{3}{*}{$\mathbf{T}_{25}$} & Untreated control & - & $0.00(0.29)$ & $0.00(0.29)$ & $0.00(0.29)$ & $0.00(0.29)$ \\
\hline & & S.Em \pm & 1.90 & 1.19 & 1.48 & 1.25 \\
\hline & & $\operatorname{CD}(p=0.01)$ & 4.49 & 4.70 & 5.83 & 4.94 \\
\hline
\end{tabular}

DAS - Day after spray Figures in the parentheses are arc sine transformed values Means followed by same alphabet do not differ significantly by DMRT $(p=0.05)$ 
Table.7 Laboratory evaluation of different insecticides and biorationals with FORS against fourth instar scale, $H$. imbricans (Pooled)

\begin{tabular}{|c|c|c|c|c|c|c|}
\hline \multirow[t]{2}{*}{ Tr. No. } & \multirow[t]{2}{*}{ Treatments } & \multirow{2}{*}{$\begin{array}{l}\text { Dosage } \\
(\mathrm{g} / \mathrm{ml} / \mathrm{l})\end{array}$} & \multicolumn{4}{|c|}{ Per cent mortality } \\
\hline & & & 1 DAS & 3 DAS & 5 DAS & 7 DAS \\
\hline $\mathbf{T}_{1}$ & Buprofezin $25 \mathrm{SC}$ & 1.25 & $41.50(40.10) b c$ & $67.50(55.24) \mathrm{a}$ & $82.00(64.89) \mathrm{a}$ & $85.00(67.21) b c$ \\
\hline $\mathbf{T}_{2}$ & Thiamethoxam $25 \mathrm{WG}$ & 0.3 & $21.50(27.62) \mathrm{e}-\mathrm{g}$ & $24.50(29.66) \mathrm{de}$ & $27.50(31.62) \mathrm{de}$ & $30.00(33.21) \mathrm{e}-\mathrm{g}$ \\
\hline $\mathbf{T}_{3}$ & Acetamiprid 20 SP & 0.2 & $19.00(25.83) \mathrm{g}$ & $19.50(26.18) \mathrm{g}$ & $23.50(28.98)$ ef & $28.50(32.25) \mathrm{fg}$ \\
\hline $\mathbf{T}_{4}$ & Imidacloprid 17.8 SL & 0.3 & $18.50(25.47) \mathrm{g}$ & $20.00(26.56) \mathrm{g}$ & $22.50(28.31) \mathrm{f}$ & $25.50(30.32) \mathrm{g}$ \\
\hline $\mathbf{T}_{5}$ & Dimethoate $30 \mathrm{EC}$ & 1.7 & $20.50(26.91) \mathrm{fg}$ & $21.50(27.62) \mathrm{fg}$ & $23.00(28.65) \mathrm{f}$ & $27.50(31.62) \mathrm{fg}$ \\
\hline $\mathbf{T}_{6}$ & Chlorpyriphos $20 \mathrm{EC}$ & 2.0 & $43.00(40.97) b c$ & $57.50(49.31) \mathrm{b}$ & $73.50(59.01) b$ & $82.00(64.89) \mathrm{c}$ \\
\hline $\mathbf{T}_{7}$ & Acephate $75 \mathrm{SP}$ & 1.0 & $45.00(42.12) \mathrm{ab}$ & $66.50(54.63) \mathrm{ab}$ & $73.50(59.01) b$ & $86.00(68.02) \mathrm{a}$ \\
\hline $\mathbf{T}_{8}$ & Deltamethrin $2.8 \mathrm{EC}$ & 0.5 & $21.00(27.27) \mathrm{fg}$ & $22.50(28.31)$ ef & $27.50(31.62) \mathrm{de}$ & $30.50(33.52) \mathrm{cf}$ \\
\hline $\mathbf{T}_{9}$ & Buprofezin 5.65 SC + Deltamethrin 0.72 EC & 2.5 & $20.00(26.55) \mathrm{fg}$ & $22.50(28.31)$ ef & $30.00(33.21) \mathrm{cd}$ & $33.50(35.36) \mathrm{e}$ \\
\hline $\mathbf{T}_{10}$ & Lambda-cyhalothrin 5 EC & 0.5 & $30.00(33.20) \mathrm{d}$ & $31.50(34.13) \mathrm{c}$ & $32.50(34.74) \mathrm{c}$ & $38.00(38.05) \mathrm{d}$ \\
\hline $\mathbf{T}_{11}$ & Methomyl 40 SP & 0.3 & $25.50(30.32) \mathrm{e}$ & $29.50(32.89) \mathrm{c}$ & $31.50(34.14) \mathrm{cd}$ & $39.50(38.93) \mathrm{d}$ \\
\hline $\mathbf{T}_{12}$ & Profenophos $50 \mathrm{EC}$ & 2.0 & $40.00(39.23) \mathrm{c}$ & $57.50(49.31) b$ & $75.00(60.00) \mathrm{b}$ & $86.00(68.02) \mathrm{a}$ \\
\hline $\mathbf{T}_{13}$ & Bifenthrin $10 \mathrm{EC}$ & 0.5 & $24.00(29.32) \mathrm{cf}$ & $25.50(30.32) \mathrm{d}$ & $30.00(33.20) \mathrm{cd}$ & $33.50(35.36) \mathrm{e}$ \\
\hline $\mathbf{T}_{14}$ & Dichlorvos $76 \mathrm{EC}$ & 1.0 & $48.00(43.85) \mathrm{a}$ & $69.00(56.17) \mathrm{a}$ & $75.00(60.00) b$ & $82.00(64.89) \mathrm{c}$ \\
\hline $\mathbf{T}_{15}$ & Lecanicillium lecani $\mathrm{WP}+$ tween 80 & $2+0.5$ & $9.50(17.95) \mathrm{h}$ & $10.00(18.43) \mathrm{jk}$ & 11.25 (19.59) h-j & $12.00(20.26) \mathrm{j}$ \\
\hline $\mathbf{T}_{16}$ & M. anisopliae WP + tween 80 & $2+0.5$ & $10.50(18.90) \mathrm{h}$ & $12.00(20.26)$ hi & $13.00(21.13) \mathrm{g}-\mathrm{i}$ & $15.50(22.78) \mathrm{ij}$ \\
\hline $\mathbf{T}_{17}$ & Mealykill & 10.00 & $9.50(17.95) \mathrm{h}$ & $12.50(20.70) \mathrm{h}$ & $15.50(23.18) \mathrm{g}$ & $17.00(24.35)$ hi \\
\hline $\mathbf{T}_{18}$ & Neemazal 15000 ppm & 5.00 & $6.00(14.17) \mathrm{i}$ & $10.50(18.90) \mathrm{ij}$ & $12.50(20.70) \mathrm{g}-\mathrm{i}$ & $14.50(22.38) \mathrm{ij}$ \\
\hline $\mathbf{T}_{19}$ & Pongamia oil + tween 80 & $10 \%+0.5$ & $6.50(14.77) \mathrm{i}$ & $8.50(16.95) \mathrm{k}$ & 9.00 (17.45) j & $20.50(26.92) \mathrm{h}$ \\
\hline $\mathbf{T}_{20}$ & Cotton seed oil + tween 80 & $10 \%+0.5$ & $9.50(17.95) \mathrm{h}$ & $10.00(18.43) \mathrm{jk}$ & $12.50(20.70) \mathrm{g}-\mathrm{i}$ & $15.50(23.18) \mathrm{ij}$ \\
\hline $\mathbf{T}_{21}$ & Sunflower oil + tween 80 & $10 \%+0.5$ & $9.00(17.45) \mathrm{h}$ & $10.00(18.43) \mathrm{jk}$ & $10.50(18.90) \mathrm{ij}$ & $12.00(20.26) \mathrm{j}$ \\
\hline $\mathbf{T}_{22}$ & Fish oil rosin soap & 5.00 & $10.00(18.43) \mathrm{h}$ & $12.50(20.70) \mathrm{h}$ & $14.50(22.38) \mathrm{gh}$ & $15.00(22.79) \mathrm{ij}$ \\
\hline $\mathbf{T}_{23}$ & Arka suraksha (IIHR Product) & 15.00 & $10.00(18.43) \mathrm{h}$ & $13.50(21.55) \mathrm{h}$ & $25.50(30.32) \mathrm{ef}$ & $17.50(24.72)$ hi \\
\hline $\mathbf{T}_{24}$ & Arka rakshak (IIHR Product) & 15.00 & $10.50(18.90) \mathrm{h}$ & $13.00(21.13) \mathrm{h}$ & $14.00(21.97) \mathrm{gh}$ & $16.00(25.10)$ hi \\
\hline $\mathbf{T}_{25}$ & Untreated control & - & $0.00(0.29)$ & $0.00(0.29)$ & $0.00(0.29)$ & $0.00(0.29)$ \\
\hline & & S.Em \pm & 1.11 & 0.78 & 1.21 & 1.17 \\
\hline & & $\mathrm{CD}(\mathrm{p}=\mathbf{0 . 0 1})$ & 4.37 & 3.08 & 4.76 & 4.01 \\
\hline
\end{tabular}

DAS - Day after spray

Figures in the parentheses are arc sine transformed values

Means followed by same alphabet do not differ significantly by DMRT ( $\mathrm{p}=0.05$ 
Table.8 Laboratory evaluation of different insecticides and biorationals without FORS against fourth instar scale, $H$. imbricans (Pooled)

\begin{tabular}{|c|c|c|c|c|c|c|}
\hline \multirow{2}{*}{$\begin{array}{l}\text { Tr. } \\
\text { No. }\end{array}$} & \multirow[t]{2}{*}{ Treatments } & \multirow{2}{*}{$\begin{array}{l}\text { Dosage } \\
(\mathrm{g} / \mathrm{ml} / \mathrm{l})\end{array}$} & \multicolumn{4}{|c|}{ Per cent mortality } \\
\hline & & & 1 DAS & 3 DAS & 5 DAS & 7 DAS \\
\hline $\mathbf{T}_{1}$ & Buprofezin 25 SC & 1.25 & $35.50(36.57) \mathrm{a}$ & $50.50(45.28) \mathrm{a}$ & $71.50(57.77) \mathrm{a}$ & $82.00(64.92) b$ \\
\hline $\mathbf{T}_{2}$ & Thiamethoxam $25 \mathrm{WG}$ & 0.3 & $20.00(26.56)$ ef & $23.00(28.65) \mathrm{cd}$ & $25.50(30.32) \mathrm{c}-\mathrm{e}$ & $27.50(31.62) \mathrm{d}-\mathrm{f}$ \\
\hline $\mathbf{T}_{3}$ & Acetamiprid $20 \mathrm{SP}$ & 0.2 & $18.00(25.09) \mathrm{f}-\mathrm{h}$ & $19.00(25.84) \mathrm{de}$ & $22.00(27.97) \mathrm{e}-\mathrm{g}$ & $27.50(31.60) \mathrm{d}-\mathrm{f}$ \\
\hline $\mathbf{T}_{4}$ & Imidacloprid 17.8 SL & 0.3 & $18.00(25.10) \mathrm{f}-\mathrm{h}$ & $19.00(25.83) \mathrm{de}$ & $21.00(27.26) \mathrm{e}-\mathrm{g}$ & $25.00(30.00)$ e-h \\
\hline $\mathbf{T}_{5}$ & Dimethoate $30 \mathrm{EC}$ & 1.7 & $20.00(26.56)$ ef & $21.50(27.62) \mathrm{cd}$ & $24.00(29.33) \mathrm{d}-\mathrm{f}$ & $26.50(30.98) \mathrm{d}-\mathrm{f}$ \\
\hline$T_{6}$ & Chlorpyriphos $20 \mathrm{EC}$ & 2.0 & $32.50(34.75) \mathrm{ab}$ & $50.50(45.28) \mathrm{a}$ & $68.50(55.86) \mathrm{a}$ & $87.00(68.92) \mathrm{a}$ \\
\hline $\mathbf{T}_{7}$ & Acephate 75 SP & 1.0 & $30.50(33.52) a-c$ & $48.50(44.14) \mathrm{a}$ & $68.50(55.86) \mathrm{a}$ & $85.50(67.64) a b$ \\
\hline $\mathbf{T}_{8}$ & Deltamethrin 2.8 EC & 0.5 & $19.50(26.20)$ ef & $21.00(27.25) \mathrm{cd}$ & $26.50(30.95) b-e$ & $29.00(32.56) \mathrm{de}$ \\
\hline $\mathbf{T}_{9}$ & Buprofezin 5.65 SC + Deltamethrin 0.72 EC & 2.5 & $19.00(25.84) \mathrm{e}-\mathrm{g}$ & $21.00(27.26) \mathrm{cd}$ & $29.00(32.57) b-d$ & $32.50(34.75) \mathrm{cd}$ \\
\hline $\mathbf{T}_{10}$ & Lambda-cyhalothrin $5 \mathrm{EC}$ & 0.5 & $28.00(31.91) b-d$ & $29.00(32.58) \mathrm{b}$ & $32.00(34.44) b$ & $36.50(37.16) \mathrm{c}$ \\
\hline $\mathbf{T}_{11}$ & Methomyl 40 SP & 0.3 & $24.50(29.66) \mathrm{c}-\mathrm{e}$ & $28.50(32.25) \mathrm{b}$ & $30.50(33.52) b c$ & $38.00(38.05) \mathrm{c}$ \\
\hline $\mathbf{T}_{12}$ & Profenophos $50 \mathrm{EC}$ & 2.0 & $32.00(34.44) \mathrm{ab}$ & $46.00(42.70) \mathrm{a}$ & $69.00(56.17) \mathrm{a}$ & $85.50(67.64) \mathrm{ab}$ \\
\hline $\mathbf{T}_{13}$ & Bifenthrin $10 \mathrm{EC}$ & 0.5 & $22.50(28.28) \mathrm{d}-\mathrm{f}$ & $25.00(30.00) b c$ & $29.00(32.56) b-d$ & $32.50(34.74) \mathrm{cd}$ \\
\hline $\mathbf{T}_{14}$ & Dichlorvos $76 \mathrm{EC}$ & 1.0 & $34.50(35.96) \mathrm{ab}$ & $49.50(44.71) \mathrm{a}$ & $69.50(56.48) \mathrm{a}$ & $82.00(64.92) b$ \\
\hline $\mathbf{T}_{15}$ & Lecanicillium lecani $\mathrm{WP}+$ tween 80 & $2+0.5$ & $14.00(21.92) \mathrm{g}-\mathrm{i}$ & $16.50(23.96)$ ef & $18.00(25.09) \mathrm{gh}$ & $22.00(27.97) \mathrm{f}-\mathrm{i}$ \\
\hline $\mathbf{T}_{16}$ & M. anisopliae $\mathrm{WP}+$ tween 80 & $2+0.5$ & $12.50(20.61) \mathrm{i}$ & $14.00(21.97) \mathrm{fg}$ & $18.50(25.45) \mathrm{f}-\mathrm{h}$ & $19.00(25.84) \mathrm{i}-\mathrm{k}$ \\
\hline $\mathbf{T}_{17}$ & Mealykill & 10.00 & $14.00(21.97) \mathrm{g}-\mathrm{i}$ & $16.50(23.97)$ ef & $19.50(26.20) \mathrm{f}-\mathrm{h}$ & $20.50(26.92) \mathrm{g}-\mathrm{i}$ \\
\hline $\mathbf{T}_{18}$ & Neemazal 15000 ppm & 5.00 & $10.00(18.43) \mathrm{i}$ & $13.50(21.55) \mathrm{fg}$ & $15.00(22.79) \mathrm{hi}$ & $17.50(24.72) \mathrm{i}-\mathrm{k}$ \\
\hline $\mathbf{T}_{19}$ & Pongamia oil + tween 80 & $10 \%+0.5$ & $9.00(21.12)$ hi & $12.00(20.26) \mathrm{g}$ & $15.00(22.79)$ hi & $17.00(24.35) \mathrm{i}-\mathrm{k}$ \\
\hline $\mathbf{T}_{20}$ & Cotton seed oil + tween 80 & $10 \%+0.5$ & $10.00(18.43) \mathrm{i}$ & $12.50(20.70) \mathrm{g}$ & $13.00(21.13) \mathrm{i}$ & $14.50(22.38) \mathrm{k}$ \\
\hline $\mathbf{T}_{21}$ & Sunflower oil + tween 80 & $10 \%+0.5$ & $9.50(17.95) \mathrm{i}$ & $11.50(19.82) \mathrm{g}$ & $13.00(21.13) \mathrm{i}$ & $15.00(22.78) \mathrm{jk}$ \\
\hline $\mathbf{T}_{22}$ & Fish oil rosin soap & 5.00 & $11.50(19.60) \mathrm{i}$ & $12.00(20.26) \mathrm{g}$ & $13.00(21.13) \mathrm{i}$ & $15.50(30.32) \mathrm{e}-\mathrm{g}$ \\
\hline $\mathbf{T}_{23}$ & Arka suraksha (IIHR Product) & 15.00 & $12.50(20.61) \mathrm{i}$ & $13.50(21.55) \mathrm{fg}$ & $17.00(24.35) \mathrm{g}-\mathrm{i}$ & $19.50(26.20) \mathrm{ij}$ \\
\hline $\mathbf{T}_{24}$ & Arka rakshak (IIHR Product) & 15.00 & $12.00(20.26) \mathrm{i}$ & $14.50(22.38) \mathrm{fg}$ & $19.00(25.84) \mathrm{f}-\mathrm{h}$ & $20.00(26.56) h-j$ \\
\hline \multirow[t]{3}{*}{$\mathbf{T}_{25}$} & Untreated control & - & $0.00(0.29)$ & $0.00(0.29)$ & $0.00(0.29)$ & $0.00(0.29)$ \\
\hline & & $\mathbf{S . E m} \pm$ & 1.60 & 1.51 & 1.73 & 1.68 \\
\hline & & $\mathrm{CD}(\mathrm{p}=0.01)$ & 5.29 & 4.97 & 5.84 & 4.02 \\
\hline
\end{tabular}

DAS - Day after spray

Figures in the parentheses are arc sine transformed values

Means followed by same alphabet do not differ significantly by DMRT $(p=0.05)$ 
However, Chlorpyriphos 20 EC @ 2 ml/l found superior among the various treatments by recording 87.00 per cent mortality and was on par with, acephate $75 \mathrm{SP} @ 1 \mathrm{~g} / \mathrm{l}$ and profenophos 50 EC @ $2 \mathrm{ml} / \mathrm{l}$. The next best treatments were buprofezin 25 SC @ 1.25 ml/1, dichlorvos 76 EC @ 1 ml/1 and lambdacyhalothrin 5 EC @ 0.5 ml/l. Least mortality $(14.50 \%)$ was recorded in cotton oil + tween 80 treatment (Table 8).

Irrespective of treatments whether imposed with or without FORS similar effects were produced with respect to mortality percentage of scales with light variation for third and fourth instars. With respect to instar susceptibility, the first instars (crawlers) of $H$. imbricans were most susceptible compared to second, third and fourth instars under laboratory condition.

Among 25 insecticides and biorationals used for the management of different stages of $H$. imbricans with and without FORS $(5 \mathrm{ml} / \mathrm{l})$ insecticides viz., buprofezin 25 SC @ 1.25 ml/l, chlorpyriphos 20 EC @ 2 ml/1, acephate 75 SP @ 1 g/l, lambda-cyhalothrin 5 EC @ $0.5 \mathrm{ml} / 1$, profenophos $50 \mathrm{EC} @ 2 \mathrm{ml} / 1$ and dichlorvos 76 EC @ 1 ml/l recorded significantly higher mortality under laboratory conditions. Irrespective of whether imposed with or without FORS effects were similar with slight variation in mortality.

Among the various instars, the first instars (crawlers) and second instars had more mortality to all treatments compared to third and fourth instars. For first instar crawlers pertained significant reduction to all imposed treatments except in untreated control. This clearly indicated that crawlers were most susceptible to all imposed treatments because of their delicate, non mealy/waxy body enabling the insecticides to come in direct contact with the insect.
Hussian et al., (2012) from Pakistan evaluated the different insecticides concentrations on mango mealybug, Drosicha mangiferae under laboratory conditions. Profenofos @ $240 \mu 1 / 30 \mathrm{ml}$ showed maximum per cent mortality of 93.3 and 86.6 per cent for first and second instar which is in full confirmation with present finding with respect

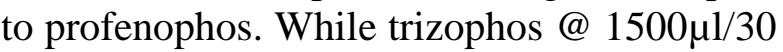
$\mathrm{ml}$ proved to be an effective insecticide for the control of fourth instar recording 64.0 and 100 per cent mortality in leaf dip method and foliar application.

Kwaiz (1999) recorded that the pre adult stages of Klifia acuminata were highly susceptible to profenofos followed by diazinon, chlorpyrifos-methyl, malathion, $\mathrm{KZ}$ and Shekrona oils compared with the adult stage. However, the efficacy of these insecticides is established the present study against all the instars of scale insect.

Present findings are also in line with Bazrafshan et al., (2010) who reported that methoxyfenozide had the least toxic effect $\left(\mathrm{LC}_{90}=30954.77 \mathrm{ppm}\right)$ and chlorpyrifos had highest toxic effect $\left(\mathrm{LC}_{90}=11636.94 \mathrm{ppm}\right)$ to the white peach scale, Pseudaulacaspis pentagona Targioni in adult stage. Based on the estimated $\mathrm{LC}_{90}$, the toxicities of all insecticides tested was rated in following order chlorpyrifos $>$ diazinon> azinphosmethyl $>$ mineral oil $>$ spinosad $>$ methoxyfenozide as being reported in the present study with respect to chlorpyrifos against $H$. imbricans.

In the present study, Neemazal 15000 ppm, Pongamia oil (10\%), Cotton seed oil (10\%) and Sunflower oil (10\%) were least effective against $H$. imbricans except for first instars. The present findings slightly deviated from the report of Hussian (1996) who reported Neem and pongamia oils at 4 per cent gave good control of various species of scales 
(Orthezia insignis Browne on crossandra and Chloropulvinaria psidii Sulz. on mango). Pongamia (4\%) and neem oil (2\% and 4\%) were found to be highly effective against the red scale on citrus (Anon., 1987). This deviation may be due to difference in scale species studied and morphology of H.imbricans in that the scale is hard like a pebble and does not allow the oils to penetrate reducing the efficacy of biorationals used in the present study.

Dhingra (1990) tested insecticidal sprays against mango mealybug, Drosicha mangiferae (Green) in the laboratory and found that lindane and malathion were ineffective. But, the LC 50 values for lambda cyhalothrin, alphamethrin, decamethrin, methyl parathion, fenvelerate, monocrotophos and endosulfan were 0.0196, 0.0209, 0.0292, $0.0385,0.0454,0.2357$ and 0.9303 per cent respectively. These results are in close agreement with present findings with respect to lambda cyhalothrin which was very effective against all four instars of H.imbricans.

The systemic insecticide monocrotophos 36 SL@ 0.08 per cent excelled over all other chemicals in managing the $P$. nigra by recording 84.04 per cent mortality, which was then followed by fenvalerate 0.02 per cent (83.45), cypermethrin 0.02 per cent (79.50), phosphamidon 0.08 per cent (79.29), dimethoate 0.08 per cent (77.93), quinalphos 0.08 per cent (76.95), phenthoate 0.08 per cent (75.00), malathion 0.1 per cent (72.30) and chlorpyriphos 0.08 per cent (69.53) (Ghule and Dhumal, 1992). These results are in line with present report with respect to efficacy of dimethoate and chlorpyriphos though species of scale insect studied is different.

Abamectin and teflubenzuron (IGR) significantly reduced the populations of $S$. oleae compared to carbaryl (Sevin 80\% WP) (Lampson and Morse, 1992). This confirms the present study with respect to effect of buprofezin. Further, Insect growth regulator (IGR) Insegar (fenoxycarb 25\%) @ $7.5 \mathrm{~g} / \mathrm{lit}$ gave better control of Saissetia oleae (Anna and Leonardi 1990) which is also in line with the present study with respect to IGR used.

Imidacloprid 17.8 per cent SL (Confidor 200 SL) at $0.01,0.05$ and 0.1 per cent a.i. on Saissetia coffeae was found effective even at 30 days after application (Irulandi et al., 2000). However, in the present study imidacloprid was less effective which may be due to change of morphology of scale species.

Dewer et al. (2012) observed that the two compounds mixtures Star oil + Admiral and Star oil + Nimbecidine were the most effective treatments against purple scale insect, Lepidosaphes beckii throughout the experiment period. Whereas the reduction percentage for Star oil + Admiral was $99.50 \%$ the reduction percentage for Star oil + Nimbecidine was $99.34 \%$, respectively. However, in the present study most of the biorationals used could not kill the different instars of $H$. imbricans which might be the incapability of these biorationals to penetrate the hard waxy/mealy cover.

\section{References}

Anna, D. R. and Leonardi, M., 1990, Preliminary tests in the control of Saissetia on citrus trees using fenoxycarb. Informatore Agrario. 47 (16) : 89-90.

Anonymous, 1987, Annu. Rep., Indian Institute of Horticultural Research, Hessaraghatta, Bangalore, p. 162.

Bazrafshan, M., Jabraeil, R., Mohammad, R., D. and Hooshang, R. D., 2010, Toxicity of several insecticides to white peach scale, Pseudaulacaspis 
pentagona Targioni (Hemiptera: Diaspididae). Mun. Ent. Zool., 5: 1020-1024.

Dewer, Y., Abdel, R. S. and Barakat, A., 2012, Comparative efficacy of some insecticides against purple scale insect, Lepidosaphes beckii (Hemiptera : Coccoidea) and its parasitoid in citrus orchard in Egypt. Egypt. Acad. J. Biol. Sci., 5 (3) : 121 127.

Dhingra, S., 1990, Alternative insecticides for the control of mango mealybugs, Drosicha mangiferae (Green) to overcome the failure of available insecticides. J. Ent. Res., 14: 139-141.

Ghule, B. D. and Dhumal, V. S., 1992, Chemical control of scale insects on pomegranate. J. Maharashtra Agric. Univ., 17 (2): 322-323.

Hussian, M. A., 1996, Evaluation of oils for the management of mealybugs and scale insects on horticultural crops. $M$. Sc. (Agri.) Thesis, Univ. Agric. Sci., Bangalore, Karnataka (India).

Hussian, S. I., Mustaq, A., Saleem, and Shoaib, F., 2012, Toxicity of some insecticides to control mango mealybug, Drosicha mangiferae, serious pest of mango from Thailand, and a key to species of the genus. Zootaxa, 1045 : 25-37.

Irulandi, S., Kumar, P. K. V., Seetharama, H. G. and Sreedharan, K., 2000, Evaluation of imidacloprid 17. 8\% SL (Confidor 200 SL) against scale insects infesting coffee seedlings, $J$. Coffee Res., 28 (1-2): 57-65

Kondo T. and Michael, L., 2005, Description of new pest species of Hemilecanium Newstead (Hemiptera: Coccidae) on mango from Thailand, and a key to species of the genus. Zootaxa, 1045: 25-37.

Kwaiz, F. A. M., 1999, Ecological and toxicological studies on the mango soft scale, Kilifa acuminata (Signoret) with special reference to insecticide residues in mango fruits. $P h . D$. Thesis, Fac. Agric. Cairo, Univ. Egypt, p. 171.

Lampson, L. J. and Morse, J. G., 1992, Impact of insect growth regulators on black scale, Saissetia oleae (Olivier) (Homoptera: Coccidae), and inter-tree dispersal. J. Agril. Ent., 9(3): 199-210.

\section{How to cite this article:}

Prakash and Patil, R.R. 2018. Evaluation of Different Insecticides and Biorationals Against Scale Insect, Hemilecanium imbricans (Green) Under Laboratory Condition. Int.J.Curr.Microbiol.App.Sci. 7(07): 866-881. doi: https://doi.org/10.20546/ijcmas.2018.707.106 